\title{
Designing a Structural Equation Model for Creative Leadership in the Relief Supply Chain in Isfahan Hospitals
}

\section{Ali Ebrahimi ${ }^{1}$}

Date of submission: 21 Jan. 2020, Date of acceptance: 22 Jul. 2020

\section{Original Article}

\begin{abstract}
INTRODUCTION: Despite the fact that natural disasters are on the rise in Iran, no study has yet explained the performance indicators in the relief supply chain in crisis situations. Creative leadership in the relief supply chain can be of great help to the enhancement of internal strengths, provision of creative solutions to potential threats, and maintenance and promotion of public health. The present study aimed to provide a Structural equation model of creative hospital leadership.

METHODS: The present exploratory research was conducted on 215 cases that were selected as the sample size using Cochran's formula. The data collection tool was a standard questionnaire which was completed by managers, supervisors, and experts of hospitals in Isfahan province. The reliability coefficient of the questionnaire was assessed using SPSS software based on Cronbach's alpha test, which indicated the high reliability of the research tool.

FINDINGS: A hierarchical structure was established using DEMATEL software, which is a variety of decision-making methods based on pairwise comparisons, and benefiting from expert opinion in extracting the factors of a system and their systematical structuring based on the principles of graph theory.

CONCLUSION: Finally, the effectiveness and affectability of the components were expressed as the output of DEMATEL software in a way that their interaction effect was denoted as numerical values. Thereafter, the data were analyzed in linear structural relations (LISREL) software using structural equation modeling, and the creative leadership model was designed.
\end{abstract}

Keywords: Creative Leadership; DEMETEL; Experts; Improvement of Relief Supply Chain.

How to cite this article: Ebrahimi A. Designing a Structural Equation Model for Creative Leadership in the Relief Supply Chain in Isfahan Hospitals. Sci J Rescue Relief 2020; 12(2): 123-134.

\section{Introduction}

$\mathrm{T}$ loday, creative leadership is considered a strategic issue, and this style of leadership can be of great help to managers in creating a good environment in crisis-oriented organizations which attracts the attention of managers of relief organizations. This attention drives from the fact that creative leaders affirm their worth in times of crisis. These leaders are expected to be qualified and competent in planning, decision-making, communication, and conflict management. Such expectations have led researchers to believe that creative leadership is a determining factor in organizational status and failure.

\section{Literature review}

Creative leadership, which is one of the styles of strategic leadership, performs a peculiar role in the promotion of strategic coordination. This style of leadership in management creates a good environment based on appropriate quality relationships and manager-employee trust. Moreover, it enhances creativity and innovation in the organization by creating a creativityfostering atmosphere, increasing individual

1- PhD in Human Resource Management, Faculty member of Bafgh Yazd Azad University, Yazd, Iran

Correspondence to: Ali Ebrahimi, Email: Ealireza88@yahoo.com 
responsibility and task transparency, as well as evaluation systems.

Adopting a creative leadership, managers enable people to come up with creative ideas to solve organizational problems. This, in turn, allows the organization to efficiently handle environmental crises which creates a proper balance between bureaucracy and freedom of action. This leadership style leads to strong intraorganizational communication that promotes a creative and innovative response system to environmental change and organizational change and adaptation (1).

This style of leadership enables leaders to discover and work on great ideas. Instead of waiting for others to develop creativity, they willingly shoulder this responsibility themselves. Creative leaders foster a culture of critical and creative thinking in organizations, they remember the past, cherish the present, and predict the future. They see new political, social, environmental, and technological situations from a broader perspective to confront present and future problems, and they firmly believe that they can build a better future (1).

Supply chain management is a set of systematic internal and external organizational activities which organizes and directs all business processes within the supply chain in a codified and holistic way in an attempt to optimize those processes at the minimum cost and maximum efficiency (2). Chegininejad and Chaharsoughi (2017) conducted a study entitled" Provide a model to explain the role of innovative marketing in supply chain leadership in order to add value to stakeholders ". They examined the role of innovative variables in value-creating leadership and its impact on value creation.

Some of the most effective parameters in this field are innovative value-creating activities, exchanged knowledge, culture, information technology infrastructure, management of values held by employees and leaders. These parameters are studied and modeled in the current research (3). Rezghi et al. (2017) conducted a study entitled "The impact of technological innovation on sustainable supply chain performance based on the company's activity type". In the mentioned research, the data were analyzed in PLS software using structural equation techniques (SEM). The results indicated that technological creativity had a significant effect on sustainable supply chain performance based on the company's activity type.

Moreover, the type of organizational activity in terms of creativity does not moderate the psychological performance of employees, environmental factors, and social effects of the performance chain (4). The related studies have demonstrated that supplier innovation has a positive effect on information sharing, strategic sourcing, and supply chain agility. Strategic sourcing also has a positive effect on supply chain agility; nonetheless, information sharing does not have a positive effect on supply chain agility (5).

Ghasemi et al. (2016) conducted a study entitled "Designing a conceptual model of innovative leadership to evaluate supply chain performance". Based on the results of the referred study, alignment with business strategy affects the evaluation of supply chain performance and efficiency. Moreover, an alliance with the power position exerts a profound effect on the evaluation of supply chain performance and efficiency (6). The term "supply chain management" was first used by management consultants in the early 1980s (7).

Organizational behavior management was initially used to explain the benefits of integrating the internal functional areas within a business, such as purchasing, manufacturing, logistics, and marketing (8). Lee et al. (2010) carried out a study entitled "Supply Chain Innovation and Organizational Performance in the Health Care Industry". In the mentioned study, in order to collect data, a Likert scale questionnaire was used and distributed in large hospitals with more than 100 beds in South Korea. The results of the stated study showed that the successful implementation of supply chain management can be achieved through continuous supply chain innovation with supplier cooperation, which in turn, improves organizational performance (9).

Wisner (2003) conducted a study entitled "Supply Chain indicators and the Relationship between Creative Leadership measures and Performance through Innovative Strategic Relationships". The results of the referred study pointed to a positive and significant relationship between innovative management measures and organizational performance (10). Yoon et al. (2016) conducted a study entitled " Effects of innovation, leadership, and supply chain innovation on supply chain efficiency: focusing 
on hospital size.

Based on the results of the mentioned study, innovation leadership is positively correlated with supply chain innovation and its dimensions (the improvement of the relief supply chain process, application of creative information technology in the relief supply chain, and relief supply chain efficiency). This, in turn, leads to an increase in supply chain efficiency, and in hospitals with more than 500 beds, innovation leadership has a positive and significant effect on supply chain innovation and its dimensions.

Furthermore, supply chain innovation has a positive and significant effect on supply chain efficiency. On the other hand, the obtained results indicated that in hospitals with less than 500 beds, the application of information technology is negatively (inversely) correlated with supply chain efficiency. Finally, it was found that supply chain innovation plays a key role in the enhancement of operational processes for supply chain efficiency. Moreover, it improves health management performance by bringing innovation to the healthcare industry (11).

The current study strived to present a creative leadership model by improving the creative process of relief chain in order to present a creative strategy in an effort to reduce the mental and physical injuries of patients in times of crisis and uninterrupted relief. Therefore, it paves the way for a theoretical framework by the revision of theoretical foundations and previously conducted studies in this field.

\section{Methods}

This study was conducted based on an exploratory-analytical, descriptive, and explanatory design to investigate the effect of variables. No article was obtained from the revision of books, scientific texts, websites, as well as scientific and research journals to help us present a structural equation model for creative hospital leadership. Therefore, the researcher used the studies conducted in the field of creative management and sociology of management regarding leadership culture and creativity.

The components extracted from these studies, which were about 480 cases, were provided to the academic experts of the Red Crescent Society using the Delphi method. Thereafter, based on the approval of components, about 71 concepts regarding creative leadership were approved, and the questionnaire was provided to five faculty members to be standardized. On a final note, according to the literature review, it was summarized to a 16 -item questionnaire. Subsequently, the structural equation model of creative hospital leadership was presented in three stages using 3 software.

After designing the model of creative hospital leadership, the components of creative hospital leadership, including the improvement of the relief supply chain process, application of creative information technology in the relief supply chain, and relief supply chain efficiency, were identified and presented as components of the model. The statistical population of the study includes 31 managers and 456 experts in hospitals in Isfahan province. Cochran's formula was used to estimate the sample size. In this formula:

$$
n=\frac{N Z^{2} p q}{N d^{2}+Z^{2} p q}=\frac{(487)(1.96)^{2}(0.5)(0.5)}{487(0.05)^{2}+(1.96)^{2}(0.5)(0.5)}=215
$$

$\mathrm{n}=$ sample size

$\mathrm{N}=$ Statistical population (including 487 managers of the Red Crescent Society)

$\mathrm{t}$ or $\mathrm{z}=$ percentage of acceptable standard error

$\mathrm{p}=\mathrm{a}$ proportion of the population without a definite attribute (e.g., male population)

$\mathrm{q}(1-\mathrm{p})=$ a proportion of the population without a definite attribute (e.g., female population)

$\mathrm{d}=$ degree of confidence

According to the above formula, the sample size with a population gap of 0.5 , signifies that half of the population have a certain trait, while the other half do not so. The value of $\mathrm{z}$ is usually 1.96. $\mathrm{d}$ can be 0.01 or 0.05 to reduce the error $\mathrm{p}=\mathrm{q}=0.5$ in the aforementioned formula, and sampling was performed at a $95 \%$ confidence interval and taking into account a 5\% error. The value of $\mathrm{p}, \mathrm{q}$ was considered to be using a $50 \%$ precautionary approach and the error of the measuring instrument is also included as 5\%.; therefore, the sample size was obtained at 215 using the mentioned formula.

\section{Information analysis method and use of Decision making trial and evaluation laboratory (DEMATEL) technique}

This research was conducted using the DEMATEL method which is a graph theorybased technique. This theory divides indicators 
into cause and effect groups to gain a better understanding of cause and effect interactions and draw a network. After the formation of the matrix, the calculation should be performed as follows:

A) Matrix normalization: The following equation is used for normalization

Equation (1):

$\boldsymbol{N}=\mathbf{z A}$

In this relation, $\mathrm{A}$ is a pairwise comparison matrix, and the value of $\mathrm{z}$ is calculated using Equation 2.

Equation (2)

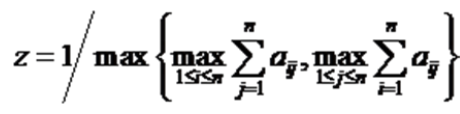

$\mathrm{N}$ is an incommensurable pairwise comparison indicator matrix.

B) Calculation of the matrix of interactions between $\mathrm{T}$-criteria using the following equation:

$$
\begin{aligned}
\boldsymbol{T} & =\boldsymbol{N}+\boldsymbol{N}^{2}+\ldots+\boldsymbol{N}^{k} \\
& =\boldsymbol{N}\left(\boldsymbol{I}+\boldsymbol{N}+\ldots+\boldsymbol{N}^{k-1}\right)\left[(\boldsymbol{I}-\boldsymbol{N})(\boldsymbol{I}-\boldsymbol{N})^{-1}\right] \\
& =\boldsymbol{N}\left(\boldsymbol{I}-\boldsymbol{N}^{k}\right)(\boldsymbol{I}-\boldsymbol{N})^{-1}=\boldsymbol{N}(\boldsymbol{I}-\boldsymbol{N})^{-1}
\end{aligned}
$$

Simply put as:

$\boldsymbol{T}=\boldsymbol{N}(\boldsymbol{I}-\boldsymbol{N})^{-1}$

Thereafter, using MATLAB software, the DIMATEL technique was coded and written in order to determine the importance and effectiveness of the components as a basis for the presentation of the structural equation model of creative leadership.

C) At this stage, firstly, the metrics in the data collection tool were used to present the model of creative leadership, and linear structural relations (LISREL) software was utilized to design the research model.

\section{Descriptive analysis of research data}

Central and dispersion indices were used for descriptive analysis of research data (Table 2).

As illustrated in the table of descriptive statistics (2), the mean values of all variables are obtained at 3 and above the average which reflects relative satisfaction with all variables with creative leadership obtaining the highest score. Moreover, the improvement of the relief supply chain process has the highest standard deviation and variance.

\section{Data normality test}

Kolmogorov-Smirnov test was used to assess the normality of the components of the model.

In all tests, the statistical hypothesis is as follows:

H0 Data are normal (data came from a normal population)

H 1 Data are not normal (data did not come from a normal population)

According to Table (3), a significant value of $>0.05$ was obtained in all cases. Therefore, there is no reason to reject the null hypothesis based on

\begin{tabular}{|c|c|c|c|}
\hline Row & Construct & Items & Cronbach's alpha coefficients \\
\hline 1 & Creative leadership & 4 & 0.86 \\
\hline 2 & Improvement of the supply chain process & 3 & 0.82 \\
\hline 3 & $\begin{array}{l}\text { The application of creative information technology in the } \\
\text { relief supply chain }\end{array}$ & 3 & 0.84 \\
\hline 4 & Relief supply chain efficiency & 6 & 0.86 \\
\hline 5 & Total questionnaire & 16 & 0.93 \\
\hline
\end{tabular}
the normality of the data.

Table 1. Reliability (validity) of the questionnaire

\begin{tabular}{|c|c|c|c|c|c|c|c|}
\hline Variable & Minimum & Maximum & Mean & SD & Variance & Skewness & Kurtosis \\
\hline Creative leadership & 1.750 & 5.000 & 3.684 & 0.589 & 0.347 & -0.259 & 0.273 \\
\hline $\begin{array}{c}\text { Improvement of relief supply chain } \\
\text { process }\end{array}$ & 1.000 & 5.000 & 3.652 & 0.759 & 0.576 & -0.840 & 1.171 \\
\hline $\begin{array}{l}\text { Application of creative information } \\
\text { technology in relief supply chain }\end{array}$ & 1.667 & 5.000 & 3.563 & 0.687 & 0.472 & -0.072 & -0.039 \\
\hline Relief supply chain efficiency & 2.000 & 5.000 & 3.584 & 0.651 & 0.424 & 0.017 & -0.575 \\
\hline
\end{tabular}

Table 2. Descriptive statistics of all research variables 


\section{Exploratory factor analysis to identify the components of a creative leadership model}

In exploratory factor analysis, the result includes three outputs. The first output shows the value of Kaiser-Meyer-Olkin (kmo), Bartlett test statistic value (which is an approximation of the Chi-square test) demonstrates the degree of freedom and significance of the test. In other words, the distribution of research data was normal and parametric tests can be performed. Since the value of kmo index was obtained at 0.746 (between 0.5-1), the number of respondents is sufficient for factor analysis.

Moreover, $p$-value of Bartlett test was less than $5 \%$ indicating the applicability of factor analysis for identifying the structure and factor model, and the assumption that the correlation matrix is known is rejected. The second factor shows the Initial subscription and the extraction intersection, respectively.

Since the initial intersection column expresses the factors before extraction, all of them are equal to 1 . When the extracted intersections are larger, the extracted factors represent the variables more effectively. When the value of the extracted intersection for the variables is small, that variable can be deleted. However, it should be noted that the deletion of variables should be performed step by step, and the variable with the least amount of extraction should be deleted in the first place.

Table 3. Names of indicators

\begin{tabular}{ccc|} 
Components & Kolmogorov-Smirnov test & Result \\
\hline Creative leadership & 0.155 & Normal \\
Improvement of the relief supply chain process & 0.094 & Normal \\
Application of creative information technology in the relief supply chain & 0.106 & Normal \\
Relief supply chain efficiency & 0.112 & Normal \\
\hline
\end{tabular}

Table 4. 71 concepts regarding creative leadership

\begin{tabular}{|c|c|c|}
\hline 699 & 1.000 & Creating a virtual network for easier access to creativity resources \\
\hline .301 & 1.000 & Improving individual creativity for the use of new technologies \\
\hline .358 & 1.000 & Creating positive feedback while working and promoting the culture of creativity to it \\
\hline .603 & 1.000 & People's involvement in creativity development \\
\hline .956 & 1.000 & Using metaphors, symbols, and patterns of creativity \\
\hline .552 & 1.000 & Focusing on people's perception of creativity \\
\hline .669 & 1.000 & Developing an outlook to promote creativity \\
\hline .945 & 1.000 & Administering creativity tests \\
\hline .496 & 1.000 & Developing a culture of creativity \\
\hline 927 & 1.000 & Motivational goals and promoting creativity in the organization \\
\hline .932 & 1.000 & Involvement of individuals in setting creative learning goals \\
\hline .927 & 1.000 & Creating a database of creativity in the organization \\
\hline .514 & 1.000 & Creating and promoting a culture of creativity in the organization \\
\hline .149 & 1.000 & Extending the influence of employee creativity in their career path \\
\hline .907 & 1.000 & Creating a culture of creativity and ways to improve it \\
\hline .166 & 1.000 & Create a creative mission outside the organization \\
\hline .043 & 1.000 & Developing creative problem-solving skills \\
\hline .042 & 1.000 & Tendency towards personal development \\
\hline .476 & 1.000 & Promoting the culture of creativity in the organization \\
\hline .907 & 1.000 & Encouraging to externalize creativity \\
\hline .950 & 1.000 & Developing the process of converting tacit knowledge into creativity \\
\hline .546 & 1.000 & Commitment of managers to the improvement of creativity \\
\hline .137 & 1.000 & Setting up a referral unit for scientific and practical problems \\
\hline .275 & 1.000 & Modification of cumbersome administrative rules regarding creativity \\
\hline .134 & 1.000 & Allocating cost to creativity as part of the cost of goods and services \\
\hline 906 & 1.000 & Creative knowledge transfer outside the organization and its internalization \\
\hline .250 & 1.000 & Collaboration with creative organizations \\
\hline .845 & 1.000 & Integrating creativity program in articles of association and strategic plan \\
\hline .229 & 1.000 & Developing a culture of learning creativity in the organization \\
\hline .262 & 1.000 & Creating a creative process for creativity-related topics \\
\hline .689 & 1.000 & Announcing the learning outcomes of people in the organization \\
\hline
\end{tabular}




\begin{tabular}{|c|c|c|}
\hline 690 & 1.000 & Using influential people for creativity \\
\hline .359 & 1.000 & Dedicating a whole day to creativity \\
\hline 638 & 1000 & Identif ving the sources of creative information to take advantage of it \\
\hline (050 & 1.000 & \\
\hline .021 & 1.000 & Applying eavesdropping styles in the organization \\
\hline .453 & 1.000 & Determining the share of organizational creativity \\
\hline .135 & 1.000 & Quantifying the value of organizational creativity \\
\hline .702 & 1.000 & Rename the training unit to the Creativity Unit \\
\hline .428 & 1.000 & Regarding organizational creativity as one of job requirements \\
\hline .887 & 1.000 & Holding creative dialogue in the organization \\
\hline .417 & 1.000 & Removing the barriers to creative attitude \\
\hline .103 & 1.000 & Linking the succession system to creativity \\
\hline .012 & 1.000 & Messages of organizational creativity in annual management reports \\
\hline .920 & 1.000 & Creating organizational memory to maintain organizational creativity knowledge \\
\hline .077 & 1.000 & Reducing middle managers for faster information flow \\
\hline .901 & 1.000 & Allowing creative knowledge to enter the organization \\
\hline .770 & 1.000 & Using coaching methods in the organization to increase creativity \\
\hline .181 & 1.000 & Organizational creativity as one of the criteria for performance evaluation \\
\hline .477 & 1.000 & Facilitating the sharing of creative knowledge in the organization \\
\hline .700 & 1.000 & Holding creativity conferences \\
\hline .227 & 1.000 & Breaking old norms in the organization \\
\hline .468 & 1.000 & Facilitating organizational relationships devoid of false bureaucracy \\
\hline .033 & 1.000 & Creating a common understanding of creativity \\
\hline .877 & 1.000 & Promoting a culture of criticism in the organization \\
\hline .561 & 1.000 & Dealing with flattery \\
\hline .759 & 1.000 & Promoting a culture of tolerance \\
\hline .727 & 1.000 & Identifying everyone's contribution to organizational creativity \\
\hline .039 & 1.000 & Creating a culture of risk tolerance in the organization \\
\hline .866 & 1.000 & Creating a common language in the organization \\
\hline .152 & 1.000 & Emphasis on quality \\
\hline .296 & 1.000 & Taking pride in superior product and service \\
\hline .732 & 1.000 & Documenting the relationship between new creative behavior and organizational success \\
\hline .912 & 1.000 & Introducing and celebrating the figures of creativity in creativity conferences \\
\hline .935 & 1.000 & Emphasis on creativity \\
\hline .535 & 1.000 & Reinforcing belief in individual abilities in the organization \\
\hline .236 & 1.000 & Negotiation \\
\hline .233 & 1.000 & Mandatory creativity \\
\hline .350 & 1.000 & Naming the organization with the pattern of organizational creativity \\
\hline .672 & 1.000 & Appropriate selection of creative people in the top positions of the organization \\
\hline .522 & 1.000 & Considering organizational creativity an intangible asset \\
\hline .375 & 1.000 & Promoting a culture of questioning \\
\hline .736 & 1.000 & Encouraging team creativity \\
\hline .254 & 1.000 & $\begin{array}{l}\text { Creating a system for acquiring knowledge and facilitating creativity } \\
\text { Extraction Method: Principal Component Analysis. }\end{array}$ \\
\hline
\end{tabular}

The third output contains three parts. The first part labeled Initial Eigenvalues, deals with eigenvalues and determines the factors that remain in the analysis (factors that have an eigenvalue of $<1$ are excluded from the analysis. Factors excluded from the analysis are the ones whose presence does not further explain the variance. The second part (Appendices), labeled as Extraction Sums of Squared Loadings, is related to the eigenvalue of extracted factors without rotation.
The hierarchical structure of 73 concepts in the system, along with the interaction among those concepts, is achieved by the judgment of experts in extracting the factors of a system and their systematic structuring based on the principles of graph theory. In so doing, the interaction effect and their importance would be determined as a numerical value. The third section (Appendices), labeled as Rotation sums of Squared Loadings, indicates the specific amount of extraction factors with rotation. 
In this analysis, five factors have a specific value of $>3$ and remain in the analysis. The main reason for choosing number 3 is the large number of variables; therefore, the number of factors can be limited in this way, and the effective variables in each factor will definitely increase. Now, the number of factors will increase significantly if this number is considered, and the effective variables in each factor will decrease in this case. Due to the reduction of influential variables in each factor, firstly, it would be difficult to name these factors. Secondly, the effect of one variable on several factors will increase, which in turn, leads to an inaccurate analysis of the effect of a variable on one factor.

Therefore, we limit the number of factors to perform the correct analysis). These five factors can explain approximately $50 \%$ of the variance of variables. It is noteworthy that in the rotation of the remaining factors, the proportion of the total changes explained by these 5 factors is almost constant. Nonetheless, contrary to the non-rotation method, in which the fifth factor explains a larger percentage of changes (approximately 50\%) in the rotation method, each factor explains approximately the same proportion of changes. This feature is the Varimax rotation that evenly distributes changes among factors.

The fourth output represents the component matrix, which contains the factor loads of each variable in the remaining factors. It is difficult to interpret factor loads without rotation; therefore, we rotate the factors to increase their interpretability. The fifth output represents the rotated component matrix which contains the factor loads of each of the variables in the factors remaining after rotation. When the absolute value of these coefficients is greater, the relevant factor is more significant in the total variation of the variable. According to factor analysis on 70 variables affecting the creativity model, three factors are identified as the main factors. These three factors should be named according to the literature review. Each factor includes the following variables:
Factor 1: Creative leadership which includes such variables as developing the creative culture of cooperative learning, creating a creative culture of trial and error and the correction methods, promoting the culture of creativity in the organization, creating a creative dialogue in the organization, using coaching methods in the organization to increase creativity, developing a culture of questioning in the organization, Setting up a referral unit for scientific and practical problems.

Factor 2: Application of information technology: It includes some variables, such as developing a clear outlook to promote creativity, administering creativity tests, extending the influence of employee creativity in their career path, developing creative problem-solving skills, the commitment of managers to the improvement of creativity, Identifying the sources of creative information, and reducing middle managers for faster information flow.

Factor 3. Relief supply chain efficiency, including such variables as the modification of cumbersome administrative rules regarding creativity, regarding organizational creativity as one of the job requirements, development of relief culture in the organization, improving individual creativity for the use of new technologies, considering organizational creativity an intangible asset, integrating creativity program into the articles of association and strategic plan.

\section{Place of DEMATEL software in designing creative leadership models}

DEMATEL, which is a type of decisionmaking methods based on pairwise comparisons, with the help of expert opinions in extracting the factors of a system and their systematic structuring based on the principles of graph theory yields a hierarchical structure of factors in the system, along with mutual effectiveness and affectability of the stated components. In so doing, it determines interaction effects and their importance as numerical values. In the current research, five indicators were used to evaluate the criteria against the target (Table 5).

Table 5. Name of indicators

\begin{tabular}{ccc|} 
Number & Symbol & Title \\
\hline 1 & $\mathrm{C}_{1}$ & Creative leadership \\
2 & $\mathrm{C}_{2}$ & Improvement of the relief supply chain process \\
3 & $\mathrm{C} 3$ & Application of creative information technology in the relief supply chain \\
4 & $\mathrm{C} 4$ & Relief supply chain efficiency \\
\hline
\end{tabular}


Table 6. Values used in the research and their equivalent names

\begin{tabular}{|cc|}
\hline Name & Value \\
\hline Effectless & 0.00 \\
Low impact & 1.00 \\
Medium impact & 2.00 \\
High impact & 3.00 \\
\hline
\end{tabular}

Moreover, six values were used to compare the criteria with each other (Table 6).

To examine the indicators, 25 experts were consulted, and Table 8 shows the pairwise comparison of each expert. In these matrices, $\boldsymbol{x}_{\boldsymbol{i} \mathbf{j}}$ is the opinion of each expert and $\boldsymbol{x}_{\boldsymbol{i} i}=(\boldsymbol{i}=$ $\mathbf{1}, \mathbf{2}, \mathbf{3}, \ldots, \boldsymbol{n}$ ) is equal to zero (The original diameter is zero) to take into account the opinion of all experts, we take their arithmetic average according to Formula 1).

Formula (1):

$$
Z=\frac{x^{1}+x^{2}+x^{3}+\ldots+x^{p}}{p}
$$

In this formula, $\mathrm{p}$ is the number of experts, and $\mathrm{x}^{1}, \mathrm{x}^{\mathrm{p}}, \mathrm{x}^{2}$ are the pairwise comparison matrix of experts 1,2 , and $p$, respectively (Table 7).

Now, it is necessary to normalize the matrices as presented in two following formulas. Formulas 2 and 3 are used to normalize the resulting matrix.

Formula (2):

$$
H_{i j}=\frac{z_{i j}}{r}
$$

$R$ is obtained using the following formula:

Formula (3):

$$
r=\max _{1 \leq i \leq n}\left(\sum_{j=1}^{n} z_{i j}\right)
$$

Therefore, the total-relation fuzzy matrix is obtained using Formula 4.

Formula (4):

$$
T=\lim _{k \rightarrow+\infty}\left(H^{1}+H^{2}+\cdots+H^{k}\right)=H \times(I-H)^{-1}
$$

In this formula, I is a unit matrix.

The next step is summing up the rows and columns of the $\mathrm{T}$ matrix. The sum of rows and columns was calculated using formulas 5 and 6 .

Formula (5):

$$
\text { (D) })_{n \times 1}=\left[\sum_{j=1}^{n} T_{i j}\right]_{n \times 1}
$$

Formula (6):

$$
(R)_{1 \times n}=\left[\sum_{i=1}^{n} T_{i j}\right]_{1 \times n}
$$

where $D$ and $R$ are $n \times 1$ and $1 \times n$ matrices, respectively. The next step is to determine the importance of the indicators $\left(D_{i}+R_{i}\right)$ and the relationship between the criteria $D_{i}+R_{i}$ (

If $D_{i}-R_{i}>0$, the relevant indicator is effective, and the relevant indicator is affectable if $D_{i}-R_{i}<0 D$. Table 7 shows $D_{i}-R_{i}$ and $D_{i}+$ $\mathrm{R}_{\mathrm{i}}$.

\section{Designing creative leadership model in the unstandardized coefficient mode}

Based on the results obtained from the output of DEMATEl software, the components were identified and the interaction effect is denoted as numerical values. Furthermore, it provides a hierarchical structure of the factors in the system, along with the effectiveness and affectability among the mentioned elements. The elements of the final structural equation model were used to measure the relationship among research constructs. The research model is presented in diagrams (1). This model is designed based on the output of LISREL software. Emotion Regulation

\begin{tabular}{|c|c|c|c|c|c|}
\hline Total-relation matrix & $\mathbf{C}_{1}$ & $\mathrm{C}_{2}$ & $\mathbf{C}_{3}$ & $\mathrm{C}_{4}$ & $\mathrm{C}_{5}$ \\
\hline $\mathrm{C}_{1}$ & 0.30 & 0.56 & 0.62 & 0.67 & 0.75 \\
\hline $\mathrm{C}_{2}$ & 0.35 & 0.29 & 0.51 & 0.56 & 0.64 \\
\hline $\mathrm{C}_{3}$ & 0.31 & 0.34 & 0.27 & 0.47 & 0.56 \\
\hline $\mathrm{C}_{4}$ & 0.29 & 0.32 & 0.35 & 0.28 & 0.53 \\
\hline
\end{tabular}
Scale: A 36-item scale designed by Graz and Roemer (2004)

Table 7. Name of indicators

\begin{tabular}{|ccc|ccc|}
\hline $\mathbf{C}_{\mathbf{5}}$ & $\mathbf{C}_{\mathbf{4}}$ & $\mathbf{C}_{\mathbf{3}}$ & $\mathbf{C}_{\mathbf{2}}$ & $\mathbf{C}_{\mathbf{1}}$ & Average of experts' opinions \\
\hline 2.60 & 2.80 & 2.81 & 2.84 & 0.00 & $\mathrm{C}_{1}$ \\
2.64 & 2.60 & 2.48 & 0.00 & 1.28 & $\mathrm{C}_{2}$ \\
2.56 & 2.13 & 0.00 & 1.24 & 1.36 & $\mathrm{C}_{3}$ \\
2.68 & 0.00 & 1.27 & 1.26 & 1.27 & $\mathrm{C}_{4}$ \\
\hline
\end{tabular}

Table 8. Total-relation fuzzy matrix 
Table 9. Importance and effectiveness of indicators

\begin{tabular}{|c|cc|}
\hline Indicator & $\boldsymbol{D}_{\boldsymbol{i}}+\boldsymbol{R}_{\boldsymbol{i}}$ & $\boldsymbol{D}_{\boldsymbol{i}}-\boldsymbol{R}_{\boldsymbol{i}}$ \\
\hline Indicator 1 & 4.40 & 1.39 \\
Indicator 2 & 4.15 & 0.56 \\
Indicator 3 & 4.02 & -0.11 \\
Indicator 4 & 4.08 & -0.54 \\
\hline
\end{tabular}

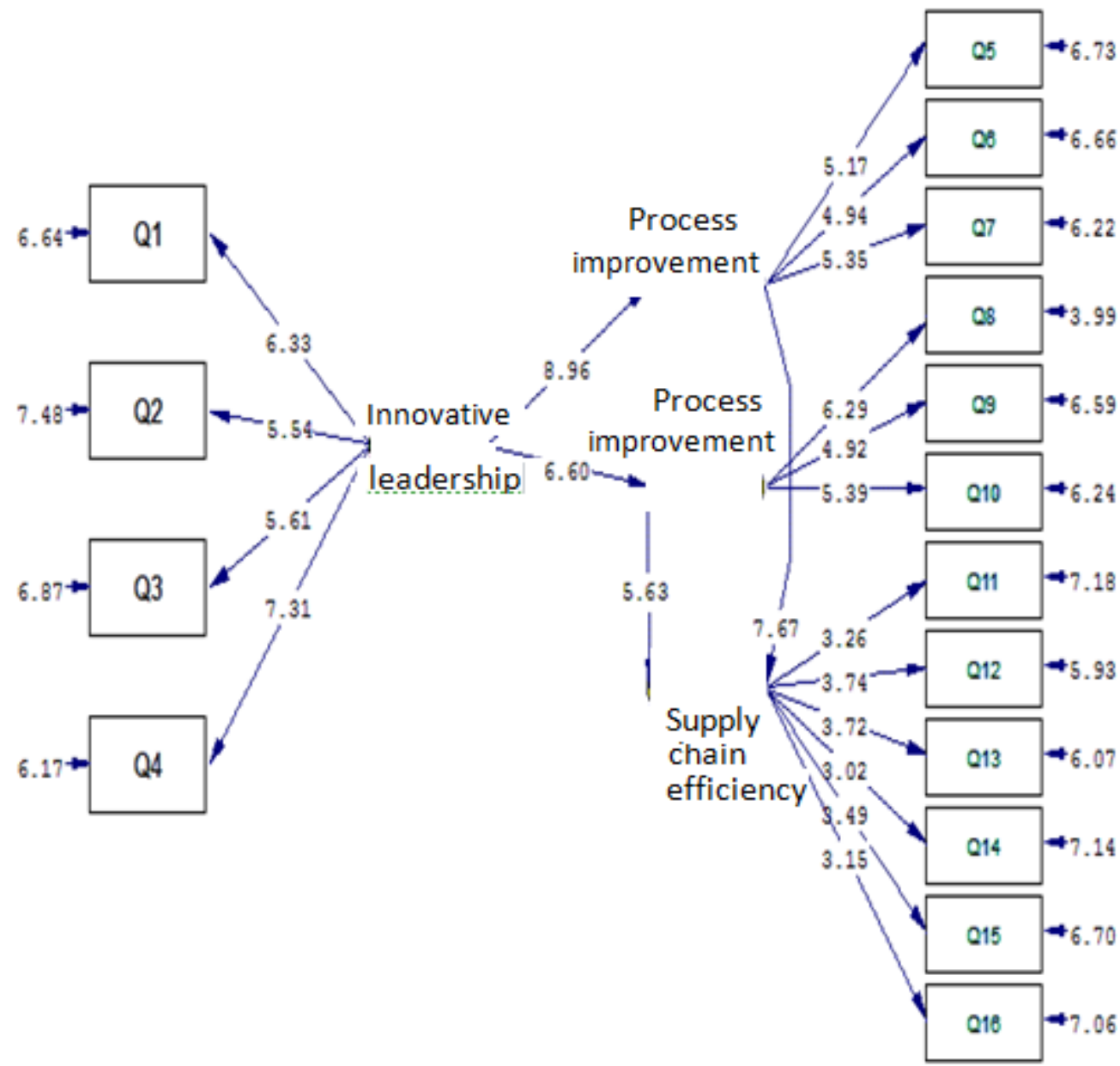

Chi-Square $=200.26, d f=100, p-$ value $=0.05648$, RMSEA $=0.035$

Diagram 1. Designing a creative leadership model in the meaningful state of research (t-value)

\section{Findings}

\section{$? ?$}

\section{Discussion and Conclusion}

The current study aimed to design and present a structural equation model for creative supply chain leadership in the Red Crescent population. The obtained findings led to the introduction of a structural equation model with a goodness of fit. In this research, in order to design a creative leadership model and work with LISREL software, obtaining model indicators alone cannot indicate its suitability or inappropriateness; nonetheless, these indicators should be interpreted jointly. Therefore, there were several fitness indicators in the present study to evaluate the model.

In the present research, to evaluate the structural equation model, X2, X2 / df, RMSEA value, as well as NFI, NNFI, IFI, AGFI, GFI indices were used. $\mathrm{X}^{2}$ test is often referred to as a success indicator. This index simply demonstrates whether the model describes the relationships among the observed variables. The smaller $X^{2}$ values are preferred. Some researchers use this ratio as an alternative index; however, this index 
has some limitations similar to $\mathrm{X}^{2}$.

There is no certainty about the ratio of chisquare to the degree of freedom and the values of $<3$ are acceptable. In the present model, it was calculated to be 2.003. The GFI index represents a relative amount of variances and covariance as explained by the model. This index varies within $0-1$, and the values closer to 1 show the better the fit of the model with the observed data. In general, in the structural equation model, when the GFI value is $>0.9$, the model is in a good position in terms of this index, the GFI value in the research model was reported as 0.97 .

Root Mean Square Error of Approximation (RMSEA) was used to investigate how the model integrates goodness of fit and savings. This index is $\leq 0.05$ for good models. The small amount of RMSEA calculated in this model (0.036) points to a suitable explanation of covariance. The goodness of fit index (GFI) was obtained at 0.92 and the adjusted goodness of fit index (AGFI) was 0.89 . When the value of these indices is closer to each other, the model fits the data better. Therefore, the proposed hypothesis model has a good fit.

\section{Factor analysis of the Creative Leadership Structural Equation Modeling Questionnaire}

The standard factor load of confirmatory factor analysis to measure the strength of the relationship between each factor (latent variable) and its observable variables (questionnaire items, including improvement of rescue and relief process, application of information technology in rescue and relief, relief supply chain efficiency) in all cases was obtained at>0.3. Therefore, the factor structure of the questionnaire can be confirmed.

After calculating the standard factor load, a significant test should be performed. Based on the results observed in the diagram below, the factor load of $t$-value of indicators in each studied dimension was $>1.96$ at a $5 \%$ confidence level.

The normal chi-square index for the above model is calculated as follows:

$$
\frac{\chi^{r}}{\mathrm{df}}=\frac{r q .1 r}{11 r}=r .1 \mathrm{NV}
$$

Moreover, since RMSEA is 0.038 , the model has a good fit. The $\mathrm{x} 2 / \mathrm{df}$ ratio does not have a fixed index for an acceptable model; nonetheless, its ideal value would be equal to 1 and must be < 3 for the acceptance of the model. The lower RMSEA index is better since the root mean index is the difference between the observed data and the model data. According to McCallum et al. (1996), < 0.1, 0.1-0.5, and 0.5-0.8 indicate excellent, good, and mediocre fit, respectively. Nonetheless, most researchers use the rule that if RMSEA $<0.1$ the fill of the model is good.

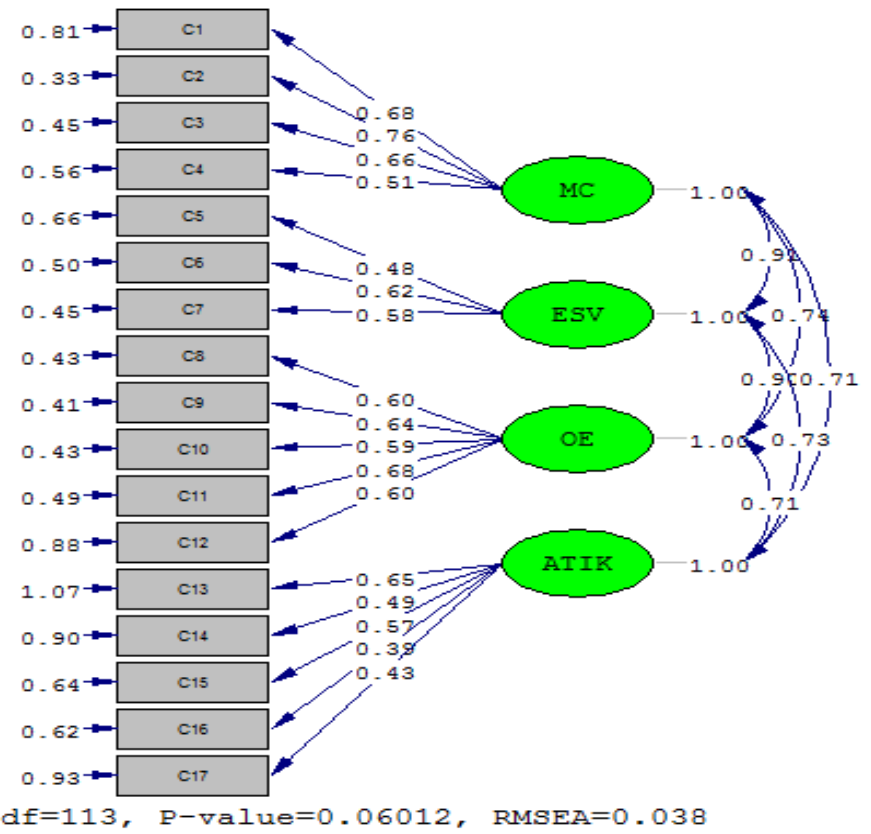

Chi-Square $=247.13, d f=113, \mathrm{P}-\mathrm{value}=0.06012, \mathrm{RMSEA}=0.038$

Diagram 2. Standard factor of the Creative Leadership Questionnaire 


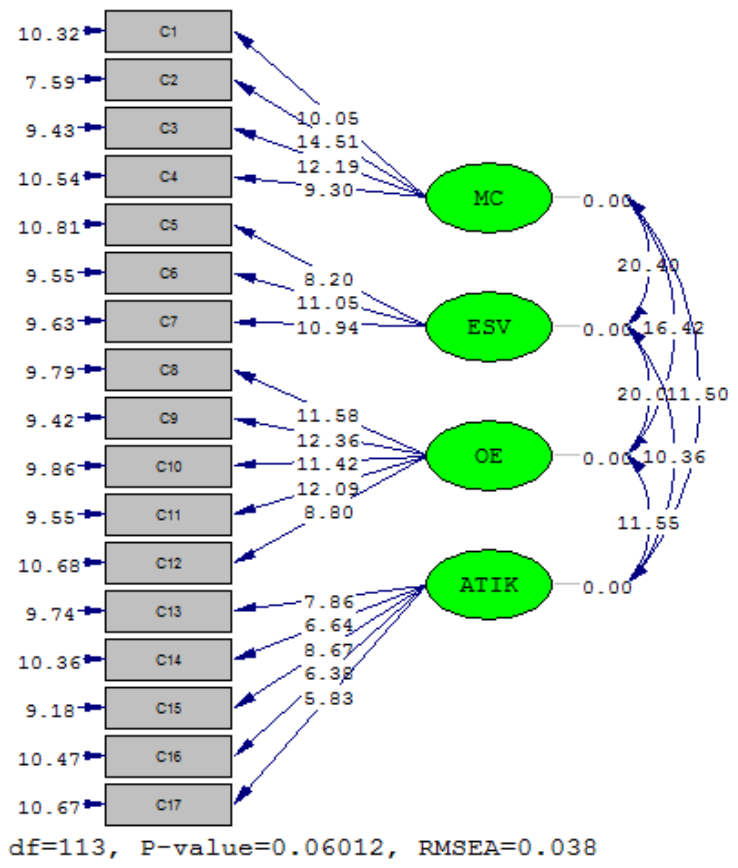

Diagram 3. T-value obtained in factor analysis of the creative leadership questionnaire

Table 10. Goodness of fit indicators of models and structural model

\begin{tabular}{|ccc|}
\hline & Indicator & Permissible limit \\
\hline RMSEA & $(\mathrm{X} 2 / \mathrm{df})$ & $<3$ \\
GFI & Root Mean Square Error of Approximation & $\leq 0.1$ \\
IFI & Goodness of fit index & $>0.9$ \\
NFI & Incremental Fit Index & $0-1$ \\
NNFI & Normal Fix Index & $>0.9$ \\
AGFI & Non Normal Fix Index & $>0.9$ \\
CFI & Adjusted Goodness of Fit & $>0.9$ \\
\hline
\end{tabular}

\section{Acknowledgments}

Our deepest appreciation goes to the editor-inchief, the officials, and referees of the Scientific Journal of Rescue and Relief who helped us in conducting this research project.

\section{Conflict of Interests}

$? ? ?$

\section{References}

1. Delafrouz et al. Supply chain management and IT support. Tadbir 2004; 15(145): 33. [In Persian].

2. Jamshidi K. Provide a model for innovative marketing in supply chain management with an information technology approach: to create higher value. [Master Thesis]. Tehran: Khajeh Nasir Toosi University of Technology; 2016. [In Persian].

3. Chegininejad D, Chaharsoughi SK. Provide a model to explain the role of innovative marketing in supply chain leadership in order to add value to stakeholders. [Master Thesis]. Tehran: Tarbiat Modares University; 2017. [In Persian].

4. Rezgi Rostami AR, Hosseini M, Asghari E, Farshidi A. The impact of technological innovation on sustainable supply chain performance based on the company's activity type. J Decis Eng 2017; 2(5): 149-66. [In Persian].

5. Kohki K, Razavi M. Investigating the impact of supplier innovation. Information sharing and strategic sourcing on improving supply chain agility. [Master Thesis]. Marvdasht: Islamic Azad University, Marvdasht Branch; 2018. [In Persian].

6. Ghasemi, B. and Valmohammadi, C. Developing a measurement instrument of knowledge management implementation in the Iranian oil industry, Kybernetes 2018; 47(10): 1874-1905. [In Persian]. https://doi.org/10.1108/K-01-20180006

7. Akman G, Yilmaz C. Innovative capability, innovation strategy and market orientation: an 
empirical analysis in Turkish software industry. Int J Innovat Manag 2008; 12(01): 69-111.

8. Henderson N, Milstein MM. Management of organizational behavior: utilizing human resources. $7^{\text {th }}$ ed. California: Prentice-Hall International Inc; 2019.

9. Marc SM, Schniederjans J. Supply chain innovation and organizational performance in the healthcare industry. Int $\mathrm{J}$ Operat Product Manag
2011; 31(1): 1193-214.

10. Shepherd C, Günter H. Measuring supply chain performance: current research and future directions. Behav Operat Plan Schedule 2010; 55(3-4): 105-21.

11. Yoon SN, Lee D, Schniederjans M. Effects of innovation leadership and supply chain innovation on supply chain efficiency: focusing on hospital size. Technol Forec Soc Change 2016; 113: 412-21. 